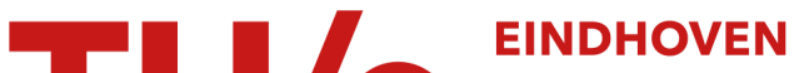 \\ UNIVERSITY OF \\ TECHNOLOGY
}

\section{Controlled synchronization via nonlinear integral coupling}

Citation for published version (APA):

Pavlov, A. V., Steur, E., \& Wouw, van de, N. (2009). Controlled synchronization via nonlinear integral coupling. In Proceedings of the 48th IEEE Conference on Decision and Control, 2009 held jointly with the 2009 28th Chinese Control Conference. CDC/CCC 2009. (pp. 5263-5268). Institute of Electrical and Electronics Engineers. https://doi.org/10.1109/CDC.2009.5400275

DOI:

10.1109/CDC.2009.5400275

Document status and date:

Published: 01/01/2009

\section{Document Version:}

Publisher's PDF, also known as Version of Record (includes final page, issue and volume numbers)

\section{Please check the document version of this publication:}

- A submitted manuscript is the version of the article upon submission and before peer-review. There can be important differences between the submitted version and the official published version of record. People interested in the research are advised to contact the author for the final version of the publication, or visit the $\mathrm{DOI}$ to the publisher's website.

- The final author version and the galley proof are versions of the publication after peer review.

- The final published version features the final layout of the paper including the volume, issue and page numbers.

Link to publication

\section{General rights}

Copyright and moral rights for the publications made accessible in the public portal are retained by the authors and/or other copyright owners and it is a condition of accessing publications that users recognise and abide by the legal requirements associated with these rights.

- Users may download and print one copy of any publication from the public portal for the purpose of private study or research.

- You may not further distribute the material or use it for any profit-making activity or commercial gain

- You may freely distribute the URL identifying the publication in the public portal.

If the publication is distributed under the terms of Article 25fa of the Dutch Copyright Act, indicated by the "Taverne" license above, please follow below link for the End User Agreement:

www.tue.nl/taverne

Take down policy

If you believe that this document breaches copyright please contact us at:

openaccess@tue.nl

providing details and we will investigate your claim. 


\title{
Controlled synchronization via nonlinear integral coupling
}

\author{
Alexey Pavlov, Erik Steur, Nathan van de Wouw
}

\begin{abstract}
This paper considers the problems of controlled synchronization and regulation of oscillatory systems. For a specific class of nonlinear systems, namely for minimum phase systems with relative degree one, we propose a systematic design procedure for finding nonlinear couplings between the systems - both unidirectional and bidirectional - that guarantee asymptotic synchronization of the systems' states for arbitrary initial conditions. The corresponding coupling has the form of an integral and it can be considered as a generalized distance between the outputs of the coupled systems. It combines both the low- and the high-gain coupling design in one nonlinear function. The results are illustrated with simulations of coupled Hindmarsh-Rose neuron oscillators.
\end{abstract}

\section{INTRODUCTION}

Synchronization of oscillatory systems and, in particular, of chaotic systems is a phenomenon that received huge attention in scientific literature for the last 15 years. The coexistence of very complex, chaotic or "irregular" dynamics of relatively simple systems on the one hand, and the possibility of some kind of "order" or synchrony in such interconnected systems, on the other hand, forms an intriguing combination for specialists in physics, mathematics, control, neuroscience and biology, thus generating a seemingly endless sequence of various results on this subject. This interest is also explained by a number of applications, already implemented or potential, of synchronization phenomena in various fields of science and technology, see, e.g., [1]-[3].

When considering synchronization phenomena in interconnected systems, one can distinguish two directions: synchronization analysis of interconnected systems with given couplings and interconnection structure, and design of interconnection couplings that guarantees systems synchronization (in a certain sense). The last problem, called the controlled synchronization problem is closely related to several control problems such as observer design and the output regulation problem, see, e.g., [4] for links between these problems. Browsing through the literature on synchronization of nonlinear systems (and synchronization in this paper is understood as asymptotic convergence of the states of the interconnected systems to each other), one encounters multiple results with linear system couplings. While for analysis of interconnected systems with given linear couplings this is a normal approach, for the problem of couplings design, limiting oneself only to linear couplings may be too restrictive, especially for highly nonlinear systems. In some cases the restriction of exploiting only linear couplings forces one to use linear high-gain designs to cope with nonlinearities in a certain set of the state space to

A. Pavlov is with StatoilHydro Research Center, Porsgrunn, No-3908, Norway. alepavestatoilhydro.com

E. Steur and $\mathrm{N}$. van de Wouw are with Department of Mechanical Engineering, Eindhoven University of Technology, P.O. Box 513, 5600 MB Eindhoven, The Netherlands. e.steuretue.nl, $\mathrm{n} \cdot \mathrm{v} \cdot \mathrm{d} \cdot$ wouwetue.nl achieve synchronization in this set [5]. At the same time, nonlinear couplings, which can be considered as couplings with varying gains, seem to be more natural for nonlinear systems: the gain should be high in the parts of the state space where the nonlinearities are essential and need to be suppressed, while it can be small in other parts of the state space. Synchronization in nonlinearly coupled systems has been considered mostly from the analysis point of view, see, e.g. [6], [7]. There are not so many results focusing on design aspects of synchronization through nonlinear coupling.

In this paper a systematic approach to the design of nonlinear coupling functions is presented for the case of two unidirectionally or bidirectionally coupled systems and for the case multiple systems interconnected via all-to-all coupling. We restrict the design to coupling functions in the form of the definite integral of some weight function with the limits being the outputs of the synchronized systems. For two systems the magnitude of the coupling can be considered as a generalized distance between the systems' outputs. For the case of a constant weight function it leads to the conventional linear coupling. The introduction of a nonlinear weight function in such an integral coupling leads to greater flexibility, which may lead to reduced coupling gains as it will be demonstrated with an example. Lower coupling gains, in turn, lead to lower (measurement) noise sensitivity. Moreover, this form of coupling is very convenient for analysis, providing neat results with constructive design methods.

This approach has its roots in the passivity-based synchronization of chaotic systems with linear couplings developed in [5] on the one hand, and recent developments in the nonlinear output regulation problem [4], [8], on the other hand. In fact, as it has been pointed out in [4], the controlled synchronization problem, at least for the case of master-slave synchronization, can be considered as a particular case of the output regulation problem. Moreover, the methods that are used both in the controlled synchronization and the output regulation problems overlap in several aspects. Controllers developed within the output regulation problem often contain the so-called internal model [9], [10] - an auxiliary dynamical system which, being a part of the controller, guarantees the existence of a solution of the closed-loop system corresponding to zero regulation error. Whether or not this solution is stabilized depends on synchrony between the system and the internal model. This synchrony is achieved by another part of the controller, usually called a stabilizer. Apart from this conceptual similarity, there is also similarity in techniques and methods employed in these problems, see, e.g., [5], [11], and the works on the convergence property [4], [12], which is the key property in terms of stabilization in both the output regulation problem and the synchronization problem.

The rest of the paper is organized as follows. The con- 
trolled synchronization problem and the proposed design method are explained in Section II. In Section III we present preliminary definitions and results on semipassivity and incremental passivity. Sections IV and V contain main theoretical results of the paper while Section VI illustrates the developed theory with an example of two nonlinearly coupled Hindmarsh-Rose oscillators. Conclusions are drawn in Section VII.

\section{CONTROLLED SYNCHRONIZATION PROBLEM}

In this paper we will consider systems of the form

$$
\begin{aligned}
\dot{x}_{i} & =f\left(x_{i}, u_{i}\right), \quad i=1, \ldots, k, \\
y_{i} & =h\left(x_{i}\right), \quad x_{i} \in \mathbb{R}^{n}, y_{i}, u_{i} \in \mathbb{R},
\end{aligned}
$$

which satisfy standard assumptions on existence and uniqueness of solutions and exhibit, for zero inputs, some bounded oscillatory dynamics. The problem of controlled synchronization of such systems that will be studied in this paper is to find coupling functions

$$
u_{i}=G_{i}\left(y_{1}, \ldots, y_{k}\right), \quad i=1, \ldots k
$$

that interconnect $k$ identical systems of the form (1) such that for arbitrary initial conditions $x_{1}(0), \ldots x_{k}(0)$ all solutions of the closed-loop system (1), (2) are defined and bounded on the infinite interval of existence, and asymptotically synchronize, i.e.

$$
\left|x_{i}(t)-x_{j}(t)\right| \rightarrow 0, \quad \text { as } t \rightarrow \infty, \quad \forall i, j .
$$

Moreover, it is required that for identical outputs $y_{1}=y_{2}=$ $\ldots=y_{k}=y$ the coupling functions must vanish,

$$
G_{i}(y, \ldots, y)=0, \quad i=1, \ldots, k,
$$

thus allowing for the $k$ interconnected systems to exhibit, in exact synchrony, the oscillatory dynamics of the original unforced system: (1) with $u_{i}=0$.

We will begin with a particular case of this problem corresponding to synchronization of two interconnected systems (1). In this case we propose a coupling in the following integral form

$$
u_{1}=\int_{y_{1}}^{y_{2}} \lambda(s) d s, \quad u_{2}=\int_{y_{2}}^{y_{1}} \lambda(s) d s
$$

with some weight function $\lambda(s)$. For a class of nonlinear systems, we will propose a design procedure to find the weight function $\lambda(s)$ that will guarantee asymptotic synchronization of the coupled systems' states for arbitrary initial conditions.

Notice that for a constant function $\lambda(s)=\lambda$, integral coupling (5) becomes the linear coupling $u_{1}=\lambda\left(y_{2}-y_{1}\right)$, $u_{2}=\lambda\left(y_{1}-y_{2}\right)$. Linear coupling has the benefit that it is simple and uniform over various values of $y_{1}$ and $y_{2}$. Yet, the systems' nonlinearities are not the same throughout the state space and for some values of the outputs $y_{1}$ and $y_{2}$ one could use a lower gain $\lambda$ than for the other values and still achieve asymptotic synchronization. The proposed integral coupling (5) overcomes the lack of versatility of the linear coupling. It allows one, through shaping the weight function $\lambda(s)$, to adjust the coupling gain depending on $y_{1}$ and $y_{2}$ (in this case the gain is understood as the ratio $\left.\int_{y_{1}}^{y_{2}} \lambda(s) d s /\left(y_{2}-y_{1}\right)\right)$. A smart choice of $\lambda(s)$ may lead to gain reduction (at least in some average sense), which, in turn may improve sensitivity of the closed-loop system to noise.

\section{PASSIVITY PRELIMINARIES}

Before presenting the main results of the paper, let us recall some definitions that will be useful for subsequent results. Consider the system

$$
\dot{x}=f(x, u), \quad y=h(x), \quad x \in \mathbb{R}^{n}, y, u \in \mathbb{R},
$$

\section{Definition 1 ( [5])}

System (6) is called $C^{1}$-semipasive if there exist a $C^{1}$ continuous nonnegative function $V: \mathbb{R}^{n} \rightarrow \mathbb{R}$ and a function $H: \mathbb{R}^{n} \rightarrow \mathbb{R}$ such that

$$
\dot{V}=\frac{\partial V}{\partial x} f(x, u) \leq y^{T} u-H(x)
$$

holds globally, where $H(x)$ is such that there exists $\rho>0$ satisfying $H(x) \geq 0$ for all $|x| \geq \rho$.

The main property of semipassive systems that will be exploited in this paper is that when interconnected through an integral coupling of the form (5) with a positive weight function $\lambda(s)$, two semipasive systems will have bounded solutions defined on an infinite interval of existence. This result is based on the ideas from [5].

\section{Lemma 1}

Consider two systems

$$
\begin{aligned}
& \dot{x}_{1}=f_{1}\left(x_{1}, u_{1}\right), \quad y_{1}=h_{1}\left(x_{1}\right), \\
& \dot{x}_{2}=f_{2}\left(x_{2}, u_{2}\right), \quad y_{2}=h_{2}\left(x_{2}\right) .
\end{aligned}
$$

Suppose both systems are $C^{1}$-semipassive with radially unbounded storage functions $V_{1}$ and $V_{2}$. Then all solutions of these systems interconnected with the integral coupling (5) with a nonnegative continuous weight function $\lambda(s) \geq 0, s \in$ $\mathbb{R}$, are defined and bounded on the infinite time interval $t \geq$ 0 . Moreover, there exists a radially unbounded nonnegative function $W\left(x_{1}, x_{2}\right)$ and a constant $c_{*} \geq 0$ such that for each $c \geq c_{*}$ the set $W\left(x_{1}, x_{2}\right) \leq c$ is a compact positively invariant set with respect to (8), (9).

Proof: Consider $W\left(x_{1}, x_{2}\right):=V_{1}\left(x_{1}\right)+V_{2}\left(x_{2}\right)$, which is a radially unbounded function. Then the semipassivity inequality (7) for the individual systems implies

$$
\begin{aligned}
\dot{W} & =\dot{V}_{1}+\dot{V}_{2} \leq y_{1} u_{1}-H_{1}\left(x_{1}\right)+y_{2} u_{2}-H_{2}\left(x_{2}\right) \\
& =\left(y_{1}-y_{2}\right) \int_{y_{1}}^{y_{2}} \lambda(s) d s-H_{1}\left(x_{1}\right)-H_{2}\left(x_{2}\right),(10)
\end{aligned}
$$

where we have substituted (5) for $u_{1}$ and $u_{2}$. Notice that since $\lambda(s) \geq 0$ for all $s \in \mathbb{R}$, then

$$
\left(y_{1}-y_{2}\right) \int_{y_{1}}^{y_{2}} \lambda(s) d s=-\left(y_{2}-y_{1}\right) \int_{y_{1}}^{y_{2}} \lambda(s) d s \leq 0
$$

for all $y_{1}, y_{2} \in \mathbb{R}$. Hence,

$$
\dot{W} \leq-H_{1}\left(x_{1}\right)-H_{2}\left(x_{2}\right) \leq 0, \forall\left|x_{1}\right| \geq \rho_{1},\left|x_{2}\right| \geq \rho_{2},
$$

where $\rho_{1}>0$ and $\rho_{2}>0$ are the constants from the definition of semipassivity of systems (8) and (9), respectively. Hence, since the function $W$ is radially unbounded, there exists $c_{*}>0$ such that $\dot{W}(x) \leq 0$ for all $x=\left(x_{1}^{T}, x_{2}^{T}\right)^{T}$ satisfying $W(x) \geq c \geq c_{*}$. Thus, the set $W\left(x_{1}, x_{2}\right) \leq c$ is a compact positively invariant set. This implies, see e.g. [13] 
that all solutions $x(t)=\left(x_{1}(t)^{T}, x_{2}(t)^{T}\right)^{T}$ are defined for all $t \geq 0$ and bounded.

\section{Definition 2 ( [8])}

System (6) is called $C^{1}$-incrementally passive if there exists a $C^{1}$ function $\Delta V\left(x_{1}, x_{2}\right): \mathbb{R}^{2 n} \rightarrow \mathbb{R}_{+}$such that

$$
\begin{aligned}
\Delta \dot{V} & =\frac{\partial \Delta V}{\partial x_{1}} f\left(x_{1}, u_{1}\right)+\frac{\partial \Delta V}{\partial x_{2}} f\left(x_{2}, u_{2}\right) \\
& \leq\left(y_{1}-y_{2}\right)^{T}\left(u_{1}-u_{2}\right)-\Delta W\left(x_{1}-x_{2}\right)
\end{aligned}
$$

for all $x_{1}, x_{2} \in \mathbb{R}^{n}, u_{1}, u_{2}, y_{1}=h\left(x_{1}\right), y_{2}=h\left(x_{2}\right)$ and some nonnegative continuous function $\Delta W(x) \geq 0$. System (1) is called strictly $C^{1}$-incrementally passive if $\Delta W(x)$ is positive definite.

The main property of incrementally passive systems that we will use for our purposes is formulated in the next lemma.

\section{Lemma 2}

Consider two identical systems

$$
\begin{array}{ll}
\dot{x}_{1}=\tilde{f}\left(x_{1}, v_{1}\right), & y_{1}=h\left(x_{1}\right), \\
\dot{x}_{2}=\tilde{f}\left(x_{2}, v_{2}\right), & y_{2}=h\left(x_{2}\right),
\end{array}
$$

interconnected through the integral coupling

$$
v_{1}=\int_{0}^{y_{2}} \lambda(s) d s, \quad v_{2}=\int_{0}^{y_{1}} \lambda(s) d s,
$$

where $\lambda(s) \geq 0$ for all $s \in \mathbb{R}$. Suppose the system

$$
\dot{x}=\tilde{f}(x, v), \quad y=h(x)
$$

is strictly $C^{1}$-incrementally passive and there exists a constant $c_{*}>0$ and a nonnegative radially unbounded function $W\left(x_{1}, x_{2}\right) \geq 0$ such that for each $c \geq c_{*}$ the set $W\left(x_{1}, x_{2}\right) \leq$ $c$ is compact and positively invariant with respect to the interconnected systems (13), (14), (15). Then each solution of the interconnected system (13), (14), (15) is defined and bounded on the infinite time interval $t \geq 0$ and satisfies

$$
x_{1}(t)-x_{2}(t) \rightarrow 0, \text { as } t \rightarrow+\infty .
$$

Proof: From the strict incremental passivity of system (16) we conclude (see inequality (12) ) that the time derivative of the function $\Delta V\left(x_{1}, x_{2}\right)$ along any solution $\left(x_{1}(t), x_{2}(t)\right)$ of system (13), (14), (15) satisfies

$$
\Delta \dot{V} \leq\left(y_{1}-y_{2}\right)\left(v_{1}-v_{2}\right)-\Delta W\left(x_{1}-x_{2}\right)
$$

for some positive definite function $\Delta W(x)$. Notice that since $\lambda(s) \geq 0$ in (15), we have

$$
\left(y_{1}-y_{2}\right)\left(v_{1}-v_{2}\right)=-\left(y_{1}-y_{2}\right) \int_{y_{2}}^{y_{1}} \lambda(s) d s \leq 0
$$

for all $y_{1}, y_{2}$. Hence,

$$
\Delta \dot{V} \leq-\Delta W\left(x_{1}-x_{2}\right)<0, \quad \forall x_{1}, x_{2} \in \mathbb{R}^{n}, \quad x_{1} \neq x_{2},
$$

since $\Delta W(x)$ is positive definite. Thus, applying LaSalle's invariance principle [13] to system (13), (14), (15) in any compact positively invariant set $W\left(x_{1}, x_{2}\right) \leq c$ for arbitrary $c \geq c_{*}$, we conclude that any solution of the interconnected system (13), (14), (15) is defined and bounded on the interval $t \geq 0$ and satisfies $\Delta W\left(x_{1}(t)-x_{2}(t)\right) \rightarrow 0$ as $t \rightarrow+\infty$, which, in turn, implies (17).

\section{Remark 1}

If the storage function $\Delta V\left(x_{1}, x_{2}\right)$ is taken in the form $\Delta V\left(x_{1}, x_{2}\right)=\tilde{V}\left(x_{1}-x_{2}\right)$ for some $C^{1}$ positive definite function $\tilde{V}(x)$, one can easily show that for each $c \geq c_{*}$ there exists a class $\mathcal{K} \mathcal{L}$ function $\beta_{c}(r, t)$ such that

$$
\left|x_{1}(t)-x_{2}(t)\right| \leq \beta_{c}\left(\left|x_{1}(0)-x_{2}(0)\right|, t\right)
$$

for every $x_{1}(0), x_{2}(0)$ satisfying $W\left(x_{1}(0), x_{2}(0)\right) \leq c$. In particular, if $\Delta V\left(x_{1}, x_{2}\right)=\left(x_{1}-x_{2}\right)^{T} P\left(x_{1}-x_{2}\right)$ and $\Delta W\left(x_{1}-x_{2}\right)=\left(x_{1}-x_{2}\right)^{T} R\left(x_{1}-x_{2}\right)$ for some positive definite matrices $P=P^{T}>0$ and $R=R^{T}>0$ independent of $c$, then there exist $\mu>0$ and $\nu>0$ such that any solution of (13), (14), (15) is bounded and satisfies

$$
\left|x_{1}(t)-x_{2}(t)\right| \leq \mu e^{-\nu t}\left|x_{1}(0)-x_{2}(0)\right| .
$$

As a tool to determine incremental passivity of a system, we provide the following lemma, which is a minor modification of a result from [8].

\section{Lemma 3}

Consider the system

$$
\dot{x}=\tilde{f}(x)+B v, \quad y=C x,
$$

with $x \in \mathbb{R}^{n}, y, v \in \mathbb{R}$ and function $\tilde{f}(x)$ being $C^{1}$. If there exist matrices $P=P^{T}>0$ and $R=R^{T}>0$ such that

$$
\begin{aligned}
P \frac{\partial \tilde{f}}{\partial x}(x)+\frac{\partial \tilde{f}^{T}}{\partial x}(x) P & \leq-R, \quad \forall x \in \mathbb{R}^{n}, \\
P B & =C^{T}
\end{aligned}
$$

then system (22) is incrementally passive with $\Delta V\left(x_{1}, x_{2}\right)=$ $\left(x_{1}-x_{2}\right)^{T} P\left(x_{1}-x_{2}\right)$ and $\Delta W\left(x_{1}-x_{2}\right)=\left(x_{1}-x_{2}\right)^{T} R\left(x_{1}-\right.$ $\left.x_{2}\right)$.

\section{CONTROLlED SYNCHRONiZATION OF TWO SYSTEMS}

In this section we consider the controlled synchronization problem for two nonlinear systems of the form

$$
\begin{aligned}
\dot{x}_{i} & =f\left(x_{i}\right)+B u_{i}, \\
y_{i} & =C x_{i},
\end{aligned}
$$

where $x_{i} \in \mathbb{R}^{n}, y_{i}, u_{i} \in \mathbb{R}, i=1,2, B$ and $C$ are constant matrices of appropriate dimensions and function $f(x)$ is $C^{1}$. The following theorem provides conditions on the weight function $\lambda(s)$ such that the states of the interconnected systems exponentially synchronize.

\section{Theorem 1}

Suppose that each system in (25) is $C^{1}$-semipassive and there exists a continuous function $\lambda(s) \geq 0, s \in \mathbb{R}$, such that

$$
\begin{aligned}
P \frac{\partial f}{\partial x}(x)+\frac{\partial f^{T}}{\partial x}(x) P-2 C^{T} C \lambda(C x) & <-R \quad \forall x \in \mathbb{R}^{n}, \\
P B & =C^{T} .
\end{aligned}
$$

Then all solutions of the systems (25) interconnected through integral coupling (5) with $\lambda(s)$ satisfying (26) are bounded and satisfy

$$
\left|x_{1}(t)-x_{2}(t)\right| \leq \mu e^{-\nu t}\left|x_{1}(0)-x_{2}(0)\right|,
$$


for some constant $\mu>0, \nu>0$.

Proof: Firstly, since $\lambda(s)$ is non-negative and each system in (25) is $C^{1}$-semipasive, Lemma 1 implies that there is a constant $c_{*}>0$ and a radially unbounded nonnegative function $W\left(x_{1}, x_{2}\right)$ such that the set $W\left(x_{1}, x_{2}\right) \leq c$ for any $c \geq c_{*}$ is compact and positively invariant with respect to (25), (5). Secondly, notice that the coupling (5) can be decomposed as

$$
\int_{y_{1}}^{y_{2}} \lambda(s) d s=\int_{y_{1}}^{0} \lambda(s) d s+\int_{0}^{y_{2}} \lambda(s) d s .
$$

Hence, the interconnected systems (25), (5) can be equivalently written as systems

$$
\dot{x}_{i}=\tilde{f}\left(x_{i}\right)+B v_{i}, \quad y_{i}=C x_{i}, \quad i=1,2,
$$

with $\tilde{f}(x)=f(x)+B \int_{y}^{0} \lambda(s) d s, y=C x$, interconnected through the coupling

$$
v_{1}=\int_{0}^{y_{2}} \lambda(s) d s, \quad v_{2}=\int_{0}^{y_{1}} \lambda(s) d s .
$$

Notice that

$$
\frac{\partial \tilde{f}}{\partial x}(x)=\frac{\partial f}{\partial x}(x)-B C \lambda(C x) .
$$

Since $P B=C^{T}$, condition (26) implies that $\tilde{f}(x)$ satisfies condition (23) of Lemma 3. By this lemma, system

$$
\dot{x}=\tilde{f}(x)+B v, \quad y=C x
$$

is strictly $C^{1}$-incrementally passive with $\Delta V\left(x_{1}, x_{2}\right)=$ $\left(x_{1}-x_{2}\right)^{T} P\left(x_{1}-x_{2}\right)$ and $\Delta W\left(x_{1}-x_{2}\right)=\left(x_{1}-x_{2}\right)^{T} R\left(x_{1}-\right.$ $\left.x_{2}\right)$. Applying Lemma 2 we conclude that any solution of systems (28) interconnected through (29) is defined and bounded for $t \geq 0$ and the states $x_{1}(t)$ and $x_{2}(t)$ asymptotically synchronize. In particular, due to Remark 1, the convergence is exponential, i.e. (27) holds.

In the case when one coupling function is set to zero, the result of the previous theorem remains the same and synchronization is still achieved. It corresponds to the socalled controlled master-slave synchronization, which can be also considered as a variant of the output regulation problem [4].

Finding $\lambda(s)$ that satisfies condition (26) is, in general, not an easy task. In the next result we specify a particular class of systems for which one can find an explicit formula for $\lambda(s)$ satisfying the conditions of Theorem 1 . To that end, consider the following system

$$
\begin{aligned}
& \dot{z}=q(z, y) \\
& \dot{y}=p(z, y)+u,
\end{aligned}
$$

where $y, u \in \mathbb{R}, z \in \mathbb{R}^{n-1}$ and the functions $q(z, y)$ and $p(z, y)$ are continuously differentiable. Notice that this system is of the form (25) with $x=\left[z^{T}, y\right]^{T}, f(x)=$ $\left[q^{T}, p\right]^{T}, B=\left[\mathbf{0}^{T}, 1\right]^{T}$ and $C=\left[\mathbf{0}^{T}, 1\right]$.

\section{Theorem 2}

Consider system (31). Suppose there exist $(n-1) \times(n-1)$ constant matrices $Q=Q^{T}>0$ and $S=S^{T}>0$ such that the inequality

$$
Q \frac{\partial q}{\partial z}(z, y)+\frac{\partial q^{T}}{\partial z}(z, y) Q \leq-S
$$

holds for all $z, y$. Suppose there exists a continuous function $\lambda(y) \geq 0$ satisfying

$$
\begin{aligned}
& \lambda(y) \geq \epsilon+\frac{\partial p}{\partial y} \\
& +\frac{1}{2}\left(Q \frac{\partial q}{\partial y}+\frac{\partial p^{T}}{\partial z}\right)^{T}\left(S-\epsilon I_{n-1}\right)^{-1}\left(Q \frac{\partial q}{\partial y}+\frac{\partial p^{T}}{\partial z}\right)
\end{aligned}
$$

for all $(z, y)$ and some $\epsilon>0$ satisfying

$$
S-\epsilon I_{n-1}>0
$$

where $I_{n-1}$ is the $(n-1) \times(n-1)$ identity matrix. Then $\lambda(y)$ satisfies (26).

Proof: Choose the matrices $P$ and $R$ in (26) as

$$
P:=\left[\begin{array}{cc}
Q & 0 \\
0 & 1
\end{array}\right], \quad R:=\left[\begin{array}{cc}
\epsilon I_{n-1} & 0 \\
0 & 2 \epsilon
\end{array}\right] .
$$

Notice that this $P$ satisfies the equality $P B=C^{T}$. By combining all the terms in the first inequality in (26) in the right-hand side, one can see that for the chosen $P=P^{T}>0$ and $R=R^{T}>0$ this matrix inequality is equivalent to

$$
J:=\left[\begin{array}{cc}
A & M \\
M^{T} & N
\end{array}\right] \geq 0,
$$

where

$$
\begin{gathered}
A=-Q \frac{\partial q}{\partial z}-\frac{\partial q^{T}}{\partial z} Q-\epsilon I_{n-1} \\
M=-Q \frac{\partial q}{\partial y}-\frac{\partial p^{T}}{\partial z}, \quad N=-2 \frac{\partial p}{\partial y}+2 \lambda(y)-2 \epsilon .
\end{gathered}
$$

Due to (32), inequality (35) holds if

$$
\tilde{J}:=\left[\begin{array}{cc}
S-\epsilon I_{n-1} & M \\
M^{T} & N
\end{array}\right]>0 .
$$

Recall that $\tilde{J}(z, y)$ is positive definite if and only if $S-$ $\epsilon I_{n-1}>0$ and $N-M^{T}\left(S-\epsilon I_{n-1}\right)^{-1} M>0$. The first inequality is guaranteed by (34), while the last one holds due to the choice of $\lambda(y)$ satisfying (33).

Function $\lambda(y)$ satisfying (33) can be found if the righthand side of (33) is independent of $z$ or can be bounded from above by a $y$-dependent function. Condition (32) guarantees that zero dynamics of system (31) (i.e. the $z$-dynamics) are convergent [12], which implies that for a given function $y(t)$ all solutions of the system $\dot{z}=q(z, y)$ converge to a unique bounded globally asymptotically stable steady-state solution determined only by $y(t)$. This property of the zero dynamics can be considered as a specific minimum phase property of the overall system (31).

\section{CONTROLlED SYNCHRONIZATION OF MULTIPLE SYSTEMS}

In this section we consider $k$ identical systems (1) interconnected by the all-to-all integral coupling of the form

$$
u_{i}=\sum_{j=i, j \neq i}^{k} \int_{y_{i}}^{y_{j}} \lambda(s) d s, \quad i=1, \ldots, k .
$$

The following results are counterparts of the corresponding results from the two-systems case. 


\section{Lemma 4}

Assume that each subsystem in (1) is $C^{1}$-semipassive with a radially unbounded storage function $V_{i}$. Then all solutions of the network of $k$ interconnected systems (1), (37) with $\lambda(s) \geq$ 0 are defined and bounded over the infinite time interval $t \geq 0$.

The proof of this lemma follows the same line of reasoning as the proof of Lemma 1 with the overall storage function equal to $W:=\sum_{i=1}^{k} V_{i}$.

\section{Theorem 3}

Consider $k$ identical $C^{1}$-semipassive systems (25). Suppose there exist $P=P^{T}>0$ and $R=R^{T}>0$ such that

$$
\begin{aligned}
& P \frac{\partial f}{\partial x}(x)+\frac{\partial f}{\partial x}^{T}(x) P-2(k-1) C^{T} C \lambda(C x) \leq-R, \\
& P B=C^{\top} .
\end{aligned}
$$

Then all solutions of the $k$ interconnected systems (25), (37) are bounded and satisfy

$$
\left|x_{i}(t)-x_{j}(t)\right| \leq \mu e^{-\nu t}\left|x_{i}(0)-x_{j}(0)\right|, \quad \forall i, j \in\{1,2, \ldots, k\}
$$

for some $\nu>0, \mu>0$.

Proof: Boundedness of solutions follows from Lemma 4. To prove synchronization of systems' states let us rewrite systems (25), (37) as follows:

$$
\dot{x}_{i}=\tilde{f}\left(x_{i}\right)+B v_{i}, \quad y_{i}=C x_{i}
$$

where $\tilde{f}(x)=f(x)+(k-1) B \int_{C x}^{0} \lambda(s) d s$ and

$$
v_{i}=\sum_{j=1, j \neq i}^{k} \int_{0}^{y_{j}} \lambda(s) d s
$$

Let $\quad X_{1} \quad:=\operatorname{col}\left(x_{1}, x_{1}, \ldots, x_{1}\right)$ and $X_{2} \quad:=$ $\operatorname{col}\left(x_{2}, x_{3}, \ldots, x_{k}\right)$. Consider the following incremental storage function $\Delta V=\left(X_{1}-X_{2}\right)^{T}\left(I_{k-1} \otimes P\right)\left(X_{1}-X_{2}\right)$, where $\otimes$ denotes the Kronecker product. Then

$$
\begin{aligned}
\Delta \dot{V}= & 2\left(X_{1}-X_{2}\right)^{\top}\left(I_{k-1} \otimes P\right)\left(F\left(X_{1}\right)-F\left(X_{2}\right)\right) \\
& -\sum_{i=2}^{k}\left(y_{1}-y_{i}\right)^{\top} \int_{y_{i}}^{y_{1}} \lambda(s) d s,
\end{aligned}
$$

where

$$
F\left(X_{1}\right)=\left(\begin{array}{c}
\tilde{f}\left(x_{1}\right) \\
\tilde{f}\left(x_{1}\right) \\
\vdots \\
\tilde{f}\left(x_{1}\right)
\end{array}\right), \quad F\left(X_{2}\right)=\left(\begin{array}{c}
\tilde{f}\left(x_{2}\right) \\
\tilde{f}\left(x_{3}\right) \\
\vdots \\
\tilde{f}\left(x_{k-1}\right)
\end{array}\right)
$$

Note that $-\sum_{i=2}^{k}\left(y_{1}-y_{i}\right)^{\top} \int_{y_{i}}^{y_{1}} \lambda(s) d s \leq 0$ since $\lambda(s) \geq 0$ for all $s \in \mathbb{R}$. Then it follows that (38) implies (see, e.g. [12])

$$
\begin{aligned}
& 2\left(X_{1}-X_{2}\right)^{T}\left(I_{k-1} \otimes P\right)\left(F\left(X_{1}\right)-F\left(X_{2}\right)\right) \\
& \leq-\left(X_{1}-X_{2}\right)^{T}\left(I_{k-1} \otimes R\right)\left(X_{1}-X_{2}\right)<0 .
\end{aligned}
$$

This, in turn, implies exponential convergence of $X_{2}(t)$ to $X_{1}(t)$ and concludes the proof of the theorem.

Finally, to apply Theorem 3 to systems of the form (31), one can still use Theorem 2 with the only modification that in the case of $k$ systems the right-hand side of (33) should be divided by $(k-1)$.

\section{EXAMPLE}

Let us consider the Hindmarsh and Rose oscillator, which represents a simplified model of neuron dynamics [14]:

$$
\begin{aligned}
\dot{z}_{1} & =c-d y^{2}-z_{1} \\
\dot{z}_{2} & =\varepsilon\left(m\left(y+y_{0}\right)-z_{2}\right) \\
\dot{y} & =-a y^{3}+b y^{2}+z_{1}-z_{2}+I+u,
\end{aligned}
$$

where $y, z_{1}$ and $z_{2}$ represent various states of a neuron and external stimulation is provided by input $u$. All other parameters are positive constants. Analysis of synchronization in a network of such oscillators with linear coupling is presented in [2], [15]. Let us apply the theory developed in the previous sections and find a nonlinear integral coupling (5) that guarantees global exponential synchronization of two identical oscillators (43). It is known that system (43) is semipassive [2], [15], so there is no need to verify this condition. Moreover, this system is in the form (31). Let us find $\lambda(y) \geq 0$ for the integral coupling using Theorem 2 . Condition (32) is satisfied with

$$
Q=\frac{1}{2}\left[\begin{array}{ll}
\gamma & 0 \\
0 & \eta
\end{array}\right], \quad S=\left[\begin{array}{cc}
\gamma & 0 \\
0 & \eta \varepsilon
\end{array}\right]
$$

where $\gamma$ and $\eta$ are arbitrary positive constants. The righthand side of inequality (33) is independent of $z$. Hence we can find $\lambda(y) \geq 0$ from (33):

$$
\lambda \geq \epsilon-3 a y^{2}+2 b y+\frac{1}{2}\left(\frac{(1-\gamma d y)^{2}}{\gamma-\epsilon}+\frac{(\eta \epsilon m / 2-1)^{2}}{2 \eta \varepsilon-\epsilon}\right),
$$

where $\epsilon>0$ is an arbitrary parameter satisfying $\epsilon<2 \eta \varepsilon$ and $\epsilon<\gamma$ due to (34). Since we want to minimize this gain function $\lambda(y)$ (for example, for reducing noise sensitivity), we get rid of the last component in (44) by choosing $\eta=$ $2 /(m \epsilon)$. This results in

$$
\lambda(y) \geq \epsilon-3 a y^{2}+2 b y+\frac{(1-\gamma d y)^{2}}{2(\gamma-\epsilon)} .
$$

Notice that the right-hand side of (45) is a quadratic function of $y$. If we choose if we choose $\gamma>0$ satisfying $\gamma<6 a / d^{2}$, then for all sufficiently small $\epsilon>0$ the right-hand side of (45) has a global maximum, which depends on $\gamma$. Further optimization of $\gamma$ within the set $\left(0,6 a / d^{2}\right)$ allows us to minimize this maximum. This can be done analytically, but here we have opted for using a simple MATLAB code for such an optimization. The parameter $\epsilon$ should be taken very small. After finding an optimal $\gamma$ and choosing a small $\epsilon$, we define $\lambda(y)$ as

$$
\lambda(y)=\max \left\{0, \epsilon-3 a y^{2}+2 b y+\frac{(1-\gamma d y)^{2}}{2(\gamma-\epsilon)}\right\},
$$

since $\lambda(y)$ has to be nonnegative.

For simulations we choose the following values of system parameters: $a=1, b=3, c=1, d=5, m=4, I=3.25$, $y_{0}=1.618, \varepsilon=0.005$ [15]. For these values of parameters the optimal value of $\gamma$ (for $\epsilon=0$ ) equals $\gamma=0.2$. The corresponding $\lambda(y)$ is shown in Figure 1. In fact, as follows from (45), one can also choose $\lambda(y)$ to be constant, which corresponds to conventional linear coupling. As follows from Figure 1, the lower bound for constant $\lambda$ that guarantees 


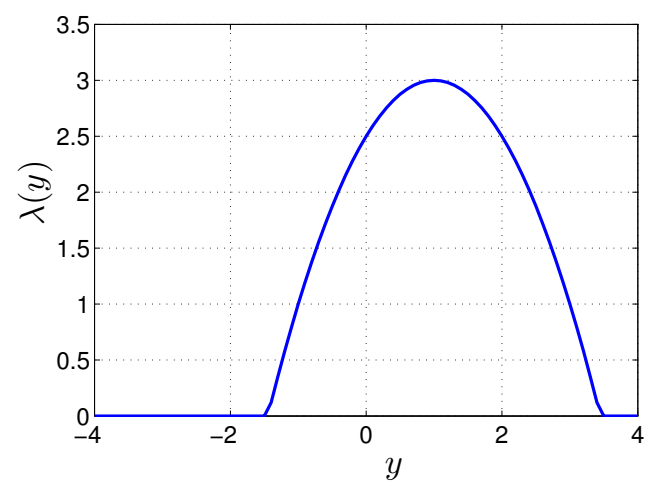

Fig. 1: Nonlinear gain function $\lambda(y)$.

global exponential synchronization equals 3 . This is already a significant improvement compared to the results from [2], where such a lower bound is computed to be equal to 10.75 (for the chosen values of parameters).

Still, the main benefit of the nonlinear integral coupling is the fact that it works like a coupling with a variable gain. It makes the gain high where necessary (e.g. where the nonlinearities are severe) and low where it is possible without compromising synchronization. This is clearly demonstrated in Figure 2, where simulation results for two coupled systems starting at the initial states $[3,0,2]^{T}$ and $[10,5,0]^{T}$ are presented. The three upper plots depict the states of the systems, while the lower plot shows the variable gain $g(t)$ of the nonlinear integral coupling defined as $g(t)=\int_{y_{1}(t)}^{y_{2}(t)} \lambda(s) d s /\left(y_{2}(t)-y_{1}(t)\right)$. The gain varies from 3 down to 0.055 . The average value of the gain over the final $700 \mathrm{~s}$ is 1.27 . In fact, in a number of simulations performed for various initial conditions, the average gain computed over intervals longer than 3000 s after synchrony had been achieved was never higher than 1.3. This value is even lower than an estimate of the lower bound for the constant coupling gain that guarantees local exponential synchronization found in [2], which equals 1.5. As follows from Figure 1, at some parts of the state space, e.g. when both $y_{1}$ and $y_{2}$ lie to the left or to the right of the parabola in Figure 1, no coupling is needed at all to maintain convergence of the system states to each other regardless of the distance between $y_{1}$ and $y_{2}$. This intriguing phenomenon has been observed in several simulations including one (with system parameters as in [2]) in which zero-coupling phenomenon occurred not only in transients, but even on the attractor. Lower gain means lower sensitivity to noise. This example demonstrates the advantages of the proposed approach to synchronization based on nonlinear integral coupling.

\section{CONCLUSIONS}

In this paper we have considered the controlled synchronization problem for a class of nonlinear systems. It has been shown that the proposed nonlinear integral coupling guarantees global exponential synchronization of two systems (either unidirectionally or bidirectionally coupled) and of $k$ systems with the all-to-all interconnection topology. A systematic procedure for finding such nonlinear couplings in the integral form is presented. The performance of the proposed method is successfully verified with simulations of two
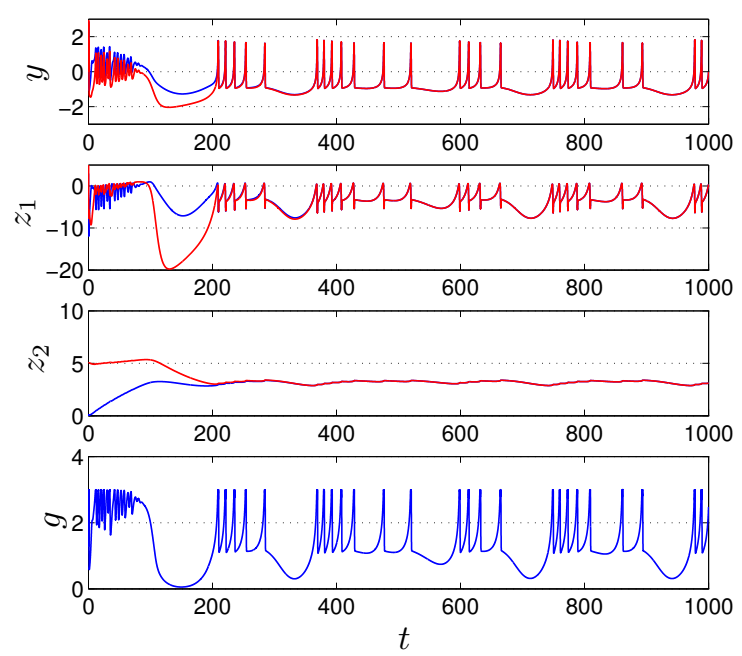

Fig. 2: Simulation results: system states and the variable gain $g(t)$.

Hindmarsh-Rose oscillators. Through this case study it has been demonstrated that the nonlinear integral coupling may lead to lower (in average) coupling gains while preserving synchronization. This, in turn, may lead to improved noise sensitivity characteristics of the overall system.

\section{REFERENCES}

[1] T. Caroll and L. Pecora, "Synchronizing chaotic circuits," IEEE Trans. Circuits Systems - I: Fundamental Theory and Applications, vol. 38, pp. 453-456, 1991.

[2] W. Oud and I. Tyukin, "Sufficient conditions for synchronization in an ensemble of Hindmarsh and Rose neurons: passivity-based approach," in Proc. of 6th IFAC Symposium on Nonlinear Control Systems, 2004.

[3] J. Levine, "On the synchronization of a pair of independent windshield wipers," IEEE Transactions on Control Systems Technology, vol. 12(5), pp. 787-795, 2004.

[4] A. Pavlov, N. van de Wouw, and H. Nijmeijer, Uniform output regulation of nonlinear systems: a convergent dynamics approach. Boston: Birkhauser, 2005.

[5] A. Pogromsky, "Passivity based design of synchronizing systems," Int. J. Bifurcation Chaos, vol. 8(2), pp. 295-319, 1998.

[6] X. Liu and T. Chen, "Synchronization analysis for nonlinearly-coupled complex networks with an asymmetrical coupling matrix," Physica A: Statistical Mechanics and its Applications, vol. 387(16-17), pp. 44294439, 2008.

[7] G. He and J. Yang, "Adaptive synchronization in nonlinearly coupled dynamical networks," Chaos, Solitons, Fractals, vol. 38(5), pp. 1254$1259,2008$.

[8] A. Pavlov and L. Marconi, "Incremental passivity and output regulation," Systems and Control Letters, vol. 57, pp. 400-409, 2008.

[9] C. Byrnes and A. Isidori, "Nonlinear internal models for output regulation," IEEE Transactions on Automatic Control, vol. 49(12), 2004.

[10] L. Marconi and L. Praly, "Uniform practical nonlinear output regulation," IEEE Transactions on Automatic Control, vol. 53(5), 2008.

[11] A. Pavlov, N. v. Wouw, and N. Nijmeijer, "Global nonlinear output regulation: Convergence-based controller design," Automatica, vol. 43(3), pp. 456-463, 2007.

[12] A. Pavlov, A. Pogromsky, N. van de Wouw, and H. Nijmeijer, "Convergent dynamics, a tribute to Boris Pavlovich Demidovich," Systems and Control Letters, vol. 52, pp. 257-261, 2004.

[13] H. Khalil, Nonlinear systems, 2nd ed. Upper Saddle River: PrenticeHall, 1996.

[14] J. Hindmarsh and R. Rose, "A model for neuronal bursting using three coupled differential equations," Proc. R. Soc. Lond., vol. B 221, pp. 87-102, 1984.

[15] E. Steur, I. Tyukin, and H. Nijmeijer, "Semi-passivity and synchronization of diffusively coupled neuronal oscillators," Physica D, Accepted, 2009. 